\title{
Smart Safety APRON for Petrochemical and Coal Mining
} \section{Industries}

\author{
Dhivya. $S^{1}$, Dharshini. $S^{2}$, Sandhiya.L ${ }^{3}$, Kavitha. $\mathrm{K}^{4}$ \\ ${ }^{1}$ Assistant Professor, \\ Department of Electronics and Instrumentation, Sri Sairam Engineering College, \\ Chennai. \\ ${ }^{2,3,4}$ UG Scholars, \\ Department of Electronics and Instrumentation, Sri Sairam Engineering College, \\ Chennai. \\ dhivya.ei@sairam.edu.in'dharshinisrivathsan@gmail.com², \\ sandhiyasandhiya1998@gmail.com ${ }^{3}$,kavithakesavankk@gmail.com ${ }^{4}$
}

\section{ABSTRACT}

In the current scenario, the safety of the coal mining and petro chemical industrial workers are not ensured as various hazardous gases are leaked and tedious health problems. Sensors are used to detect the leakage of gases. Effective monitoring of various gases and information regarding the gas leakages are not properly maintained. This proposed project aims to provide smart apron for the workers in petrochemical and coal mining industries. Various parameters like methane gas, carbon monoxide gas, hydrogen sulphide gas and even the plant temperature can be detected by different sensors. Whenever the gas reaches specific threshold value it leads to serious health problems and may even leading to explosion of the entire unit. Therefore, to avoid these issues, continuous monitoring of gas leakages is required and whenever the gas exceeds the threshold value the system immediately alerts the worker about the danger and GSM module is used to send the relevant information to the control room. Thus the effective monitoring and control of gas leakages can be performed directly from the control room and ensures the safer industrial environment.

KEYWORDS: Safety apron, Gas sensor, Hazardous gases, Arduino , GSM module, module, Buzzer.

\section{INTRODUCTION}

Modern level of electronics and communication technology allows incorporating electronic components directly into work uniforms or civilian clothes without bringing any physical discomfort to its wearer. This technology also provides remote monitoring feature, when all data about a wearer can be analyzed by competent personnel remotely and in relation with functions of wearable devices. "This work was not supported by any organization."
There are a lot of professions in industry and service sectors which are affected by different hazards. At the same time, the safety standards for industrial plants become tougher with respect to employee state monitoring and monitoring of working environmental conditions. Therefore, it is necessary to provide real time wireless monitoring of both employee state and workplace environmental conditions. That real-time monitoring can be carried out by constructing wearable sensors into 
personnel uniforms to achieve new life quality in different spheres such as medicine, safety, sports and military. From industry point of view, such "smart apron" designs provide the possibility of monitoring the employee state during the work, predicting possible health decline and preventing emergency states. Various types of sensor networks have been developed recently . These networks consist of small nodes and are equipped with GSM, microprocessors and sensors . They can be used in different areas of life for environmental and human monitoring, home automation, etc. Using sensor networks with wearable technology improves the early detection of hazardous situations and transmitting and controlling. These "smart" aprons provide a precise monitoring of workplace environmental conditions and in case of emergency states. This technology enables communication between control room and devices. In terms of using combustible gas sensors in a apron, the main problem is the high power consumption of sensor. At the lower explosive limit in the concentration range, the catalytic sensors would be widely used for the measurement of combustible gas, since it has the characteristics of highsensitivity and more selectivity, linear response and low cost. So, we have chosen a catalytic sensor for the proposed wearable sensor system. In this work, we have presented a wearable sensor system which can be designated for monitoring environmental parameters and providing employees' safety in hazardous industries such as oil and gas. The sensor system parts are attached to the employee's cloth and provide continuous data collection and transmission of data when it reaches its own threshold value. As a matter of fact, the proposed wearable sensor system is a combination of the features for the measurement of various gases in the working environment. Such a wearable system can be used in hazardous industries where it is difficult to analyse the gas leakage. The wearable system proposed in this work, provides data collection and ensures personnel safety and control of gases can also be made possible. In case of entering the remote activation zone, if the gas leakage prevails the sensors will get activated and transmit those signals to the control room.

\section{EXISTING SYSTEM}

In the current situation if any gas leakage occurs in the petrochemical or coal mining industries it has to be first sensed by the workers and this facility is made available for the workers by placing sensors at the industrial site. If they want to inform the situation to the control station then they have to use some other means by contacting them through phones or even at most worst situation for emergency indication they have to break the break glass to pull the emergency chain so that the person in the control station will come to know about the upcoming danger in the plant. This system is very critical because if there is excess amount of hazardous gas leakage occurs the workers will be quickly fainted so he cannot be able to send information to the control station. This may cause more severe problems such as explosion of the plant. And also it may cause the death of the workers who is exposed to the hazardous gases for a very long time. even a small amount of hazardous gas exposure causes more severe health problems to the workers of 
petrochemical and coal mining industries.

\section{PROPOSED SYSTEM}

This system provides the smart safety apron for the detection and control of hazardous gases in the petrochemical and coal mining industries. This system avoids hazardous environment inside the process plants. The sensors provided here will helps the operator to know about the leakages of any hazardous gases in the plant.A small amount of hazardous gas leakages causes the severe health issues and even the explosions may occur.An GSM module is also can be used to send the information to the control station about any gas leakages inside the plant. An buzzer is provided along with the apron so as to indicate the workers about the upcoming dangers. This system helps the workers by not getting affected by the hazardous gases and the accidents can also be avoided.This system can be further modified in future that for the detection of gases in the plant areas before entering to it by using the plc techniques which can be controlled from the control room itself.

\section{WORKING}

The various gases like methane, carbon monoxide, hydrogen sulphide can be detected using the gas sensor (MQ6), (3SP) are connected to the Arduino which senses and given an alarm when the leakage prevails. If the gas value exceeds the particular threshold value a voice alert and the message alert may be given to the control room through the transmitting GSM module. From the control room through the receiving GSM when the gas reaches threshold value it can be controlled and monitored. This serves as a easy platform to monitor and control the gases and also safeguards the industrial environment. This wearable device can be used in the petrochemical and coal mining industries.

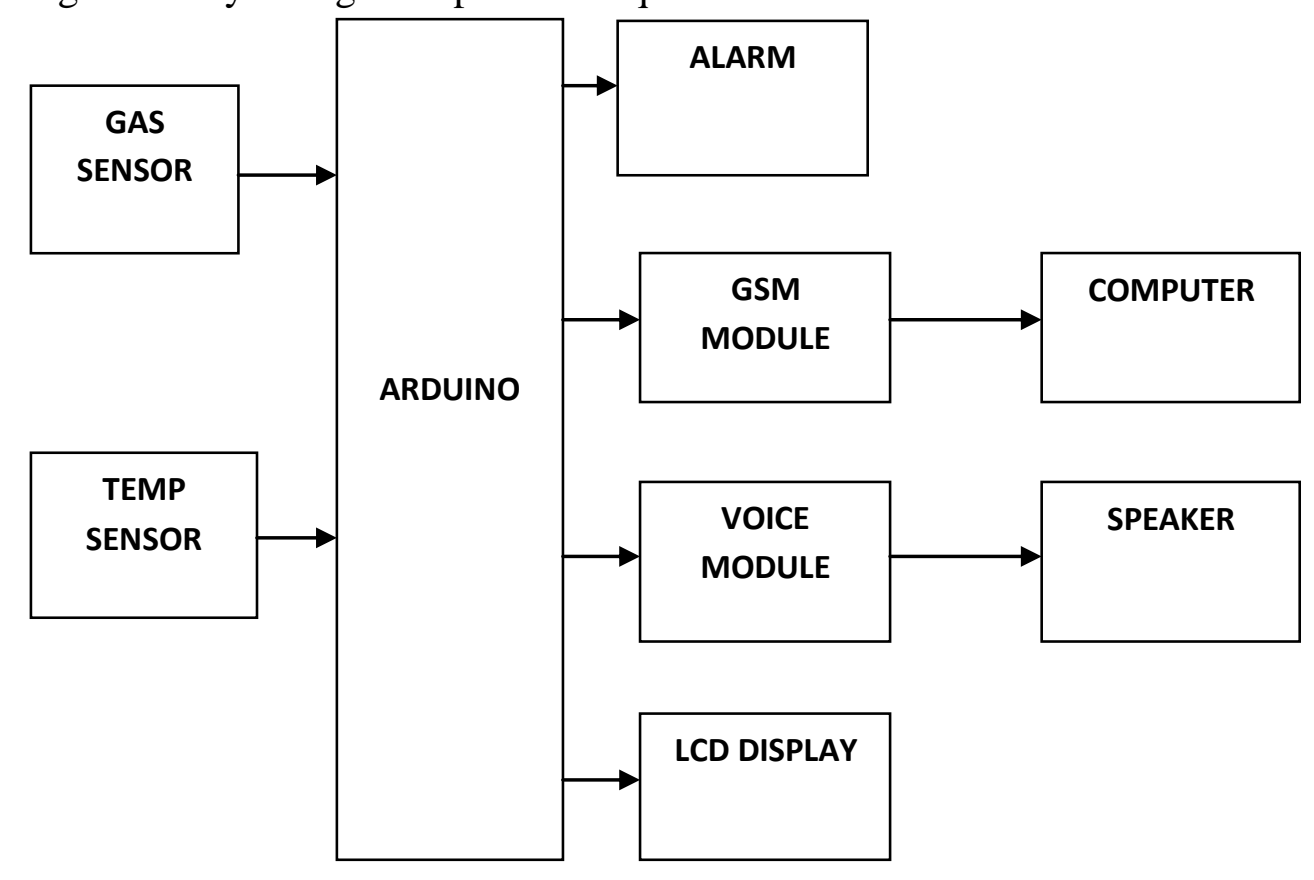

Fig:1-BLOCK DIAGRAM 


\section{SYSTEM OVERVIEW}

The block diagram of the uniform with a the smart safety apron system is illustrated .The system consists of several parts :a power source (1), a sensor module (2), GSM module(3), a light indicator (4), a buzzer (5), voice module(6) and speaker(7). Since the system is wearable and attached to clothes, all its parts are light and small. It is necessary that the system parts are placed according to their functions and distributed around the clothes in order to be worn comfortably. As for the power source of the system, normal home power supply. The sensor system monitors the atmosphere temperature as well as the concentration of combustible gases. The system consists of a light indicator and a buzzer in order to handle alarm signals and send and receive data using the by GSM module. The block diagram of the sensor module is presented below. The module is based on an AT megamicrocontroller. A commercial catalytic gas sensor is used for measuring combustible gases concentration. Since the catalytic gas sensor is very power hungry, a special measuring algorithm is used to decrease its power consumption. More detailed information concerning the measuring circuit and the algorithm is presented. The sensor module is placed on the left arm and is connected to the power supply through a cable ribbon. The module is also connected to the GSMmodule, light indicator and the buzzer and voice module. The light indicator resistor is conditions are transmitted to position on the left hand wrist in order to be on the line of sight of the wearer. It consists of a light emitting diode and a current limiting resistor. The buzzer is located on apron. Whenever it reaches or exceeds a threshold value and hazardous situation occurs the light indicator and buzzer notify the wearer by generating record voice. Except visual and audio signals, information about hazardous other devices in a GSM module network. For this purpose the transceiver module gsm dual mode ETRX3 is used. The transceiver is also used to perform employee's presence tracking. The algorithm to detect the wearer presence in power plant. If the system reaches a threshold value, the arduinoconnected with buzzer and voice module produce a alarm message from the system.

\section{FUTURE ENHANCEMENT}

This system can be further modified by connecting the LED in the apron for alerting the workers if they are busy in talking through phone or directly. When they are busy talking they cannot be able to hear the buzzer sound. So LED can be useful to indicate the fore coming dangers for the workers. Also the leakage of hazardous gases may be controlled by using PLC.

\section{CONCLUSION}

This project demonstrates a wearable sensor system which can be attached with apron and used for monitoring hazardous gas concentration and temperature. The main feature of this project is the leakage of the gases may be controlled from the control room itself which ensures the safety of the workers and prevents the plant from accidents. 


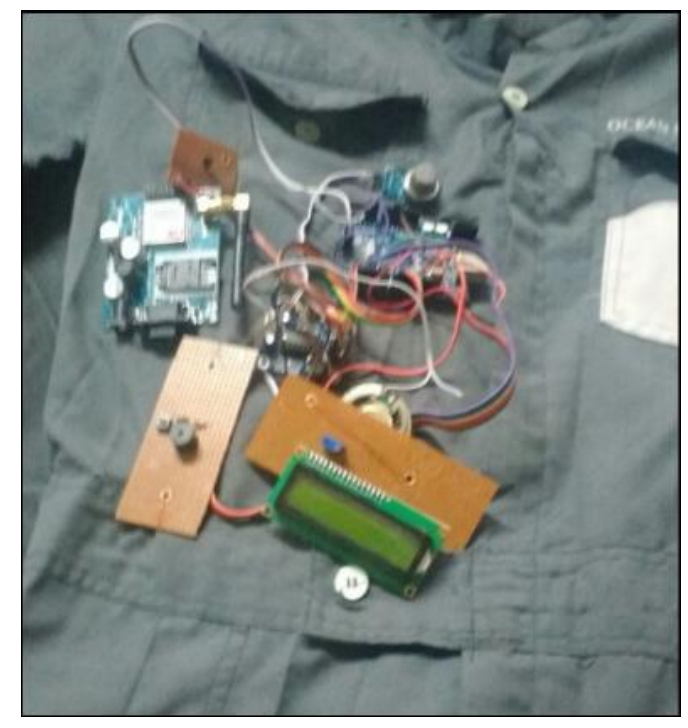

Fig:2-MODEL OF SMART SAFETY APRON

\section{REFERENCE}

1. Z Xusheng Z. Yunlong "Accident cause analysis and counter measure of coal and gas outburst nearly two years of our country" Mining Safety and Environmental Protection. vol. 37 no. 1 pp. 84-27 2010.

2. Y. Dawud "Smoke episodes and assessment of health impacts related to haze from forest fires: Indonesian experience" The Indonesian Association of Pulmonologist Persahabatan Hospital Jakarta. pp. 313-322 1999. 